Les nationalismes et la construction européenne

\title{
Les protestants en Irlande du nord face à l'Europe
}

\section{Elisabeth Picard}

\section{(2) OpenEdition}

1 Journals

\section{Édition électronique}

URL : http://journals.openedition.org/conflits/654

DOI : $10.4000 /$ conflits.654

ISSN : $1777-5345$

Éditeur :

CCLS - Centre d'études sur les conflits lilberté et sécurité, L'Harmattan

\section{Édition imprimée}

Date de publication : 22 octobre 1992

ISSN : 1157-996X

Référence électronique

Elisabeth Picard, «Les protestants en Irlande du nord face à l'Europe », Cultures \& Conflits [En ligne], 07 | automne 1992, mis en ligne le 06 janvier 2003, consulté le 30 mars 2021. URL : http:// journals.openedition.org/conflits/654; DOI : https://doi.org/10.4000/conflits.654

Ce document a été généré automatiquement le 30 mars 2021.

Creative Commons License 


\title{
Les protestants en Irlande du nord face à l'Europe
}

\author{
Elisabeth Picard
}

1 Pour la grande majorité des observateurs et des analystes de la crise en Irlande du Nord, les notions de minorité opprimée, de lutte armée et de terrorisme se rattachent à la communauté nationaliste (irlandaise) catholique. Combinées d'une façon plus ou moins ex licite, la sympathie envers la culture celtique ressentie à la fois comme authentique et comme héroïque, et l'horreur devant les violences perpétrées par l'IRA incitent fréquemment à réduire la question irlandaise à la revendication nationaliste. Il n'est pourtant que de se reporter aux données démographiques pour comprendre que la question d'Irlande du Nord est aussi (et demain, surtout?) celle de l'avenir de $60 \%$ de la population de la province : environ un million de sujets du Royaume-Uni de diverses dénominations protestantes, qui ne constituent par contre que $20 \%$ de la population totale de l'île d'Irlande, majoritairement républicaine et catholique.

2 Après cinquante années de gouvernement sans partage de l'Irlande du Nord les Unionistes protestants, qui avaient obtenu en 1920 le maintien de six des neuf comtés de l'Ulster au sein du Royaume-Uni, ont perdu en mars 1972 leur autonomie provinciale au profit d'une administration directe par Londres. Or, le début des "troubles" (c'est ainsi qu'on appelle depuis vingt-cinq ans la guerre en Irlande du Nord), l'arrivée de l'armée britannique et la reprise en main de la province sont advenus dans la période même de l'entrée simultanée du Royaume-Uni et de la république d'Irlande dans la Communauté européenne (janvier 1973). Aujourd'hui encore, dans cette année charnière 1992 où le Royaume-Uni et la république d'Irlande disent oui à Maastricht et où le Marché unique entre en vigueur, les négociations entre partis politiques du Nord ("strand I"), entre le Nord et la République du Sud ("strand 2") et entre les États concernés ("strand 3") par le conflit irlandais connaissent une accélération surprenante. Plus qu'une coïncidence, cette synchronie est bâtie sur des interactions nombreuses mais contradictoires entre le conflit civil intérieur à l'espace nordirlandais et les transformations politiques et économiques de l'espace européen. En particulier, il existe de profondes différences entre l'appréhension de l'Europe par les 
Unionistes d'Irlande du Nord, et celle de leurs concitoyens nationalistes, entre la relation des uns et des autres à la Communauté. Et ces différences sont riches d'enseignements sur le statut des États, sur la logique des groupes d'intérêt et sur la dynamique des identités nationales et infra-nationales dans la construction européenne aujourd'hui.

L'Europe, cheval de Troie

3 La méfiance, et même l'hostilité, des Unionistes nord-irlandais envers la construction européenne est l'image inversée des attentes et de la satisfaction grandissante des Nationalistes. Déjà, au début des années 1960, Ies ministres unionistes du gouvernement nord-irlandais avaient appréhendé les effets négatifs d'une entrée dans la Communauté sur la protection de l'emploi dans leur province. Au référendum de 1975 sur l'adhésion du Royaume-Uni, l'Irlande du Nord a battu tous les records de vote négatif du pays ${ }^{1}$ et encore aujourd'hui, la région reste une de celles de l'Europe où la CEE suscite le moins d'intérêt et d'enthousiasme, amplifiant en quelque sorte le syndrome insulaire britannique. Certes, des Unionistes ont été candidats aux élections parlementaires européennes de 1979, 1984 et 1989. Sur trois députés de la province au parlement de Strasbourg, deux sont aujourd'hui des Unionistes: Jim Nicholson pour le parti majoritaire (35\% des voix), l'Ulster Unionist Party, et le célèbre pasteur Paisley, fondateur du Democratic L1nionist Party. Mais tant pour l'UUP que pour Ie DUP qui persiste à proclamer "non à Maastricht, non à Delors"2, cette participation remplit deux objectifs prioritaires : d'abord, et toujours, réaffirmer les positions unionistes face aux Nationalistes et à la République du Sud ; ensuite, et surtout, contrôler la politique du Royaume-Uni sur le théâtre européen.

Pour les Unionistes, les institutions et les pratiques européennes menaçaient en effet de fournir à la République et aux Nationalistes une multiplicité d'occasions d'effacer la frontière farouchement gardée des six comtés du Nord. En réalité, cette menace est restée limitée puisque deux domaines essentiels de la souveraineté britannique en Irlande du Nord sont restés jusqu'ici à 1 abri de l'intrusion européenne et, à travers elle, de l'influence irlandaise : celui des échanges financiers ${ }^{3}$, puisque le Royaume-Uni n'est pas entré dans le système monétaire européen en 1979 ; et celui de la sécurité, puisque ni le Royaume-Uni ni l'Irlande ne sont signataires de l'accord de Schengen (1987). Mais au plan politique, l'effet redouté ne se fit pas attendre. Après s'être une première fois reconnu incompétent, le Parlement européen débattit en mars 1984 d'un rapport sur la situation en Irlande du Nord, présenté par son Comité des Affaires politiques ${ }^{4}$, en dépit des protestations véhémentes des deux députés unionistes arguant que l'Assemblée devait $s$ abstenir d'intervenir dans les affaires intérieures d'un État membre. Le rapport appelant à un dialogue intercommunautaire et surtout à une négociation entre Londres et Dublin sur la province, fut adopté par 124 voix contre 3, tandis que les députés conservateurs britanniques s'abstenaient. Quelques mois plus tard, en novembre 1985, cette même assemblée saluait avec satisfaction la signature de l'accord anglo-irlandais sur l'Irlande du Nord, accordant à la République un rôle de garant de la minorité nationaliste.

Ce que les Unionistes avaient perçu avant même 1973, c'est que la République et les Nationalistes trouveraient à Bruxelles et à Strasbourg un soutien d'autant plus chaleureux que 1 adhésion de Londres était entachée de réticences. Autant un parti nationaliste modéré comme le SDLP a ses entrées partout à Bruxelles, autant l'UUP, le DUP et la petite formation centriste Alliance s'y sentent isolés. En sujets loyaux de sa 
Majesté, les Unionistes se méfient de l'Europe. Mais celle-ci le leur rend bien. Aucun groupe politique européen, pas même celui des Chrétiens Démocrates auquel est affilié Nicholson $^{5}$, ne prend leur défense ni ne répercute dans les milieux communautaires la volonté légitime de la majeure partie de la province de rester citoyens britanniques ${ }^{6}$. Quant à Ian Paisley, par sa conduite de trublion et ses flirts avec les groupes européens d'extrême droite, il n'a pas amélioré l'image de conservateurs obsolètes et peu démocratiques qui colle aux Unionistes nord-irlandais.

Cette perception inégale suffit-elle à expliquer que, venant d'Europe, les commentaires, les recommandations et les projets de règlement de la crise nord-irlandaise font tous la part belle à Dublin et aux Nationalistes ? Ou est-ce seulement qu'il ne saurait y avoir de solution négociée à cette crise sans un rééquilibrage politique dont les Unionistes devront faire les frais? Pour ces derniers, l'affaire est malheureusement entendue : le lobby "catholique" (ou encore le "Romanish plot") est tout puissant à Bruxelles; les Unionistes arrivent toujours derrière, souvent seulement pour réagir aux initiatives nationalistes. Qui est à l'origine de l'ouverture d'un Bureau de l'Irlande du Nord à Bruxelles en février 1992 ? Le SDLP, sur la base d'une étude de faisabilité commandée à des universitaires proches du parti ${ }^{7}$. Qui est derrière les projets circulant à Bruxelles, de partage des pouvoirs dans la province entre une assemblée élue, un conseil, et un exécutif dont 4 des 6 membres seraient désignés respectivement par la CEE et par le gouvernement irlandais (et les deux derniers par le gouvernement britannique)? De hauts fonctionnaires bruxellois, anciens membres du SDLP${ }^{8}$. Et cette stratégie de mise en place d'un pouvoir régional sous le patronage communautaire ne prépare-t-elle pas en fait "la future cession de l'Ulster à l'Eiren" ?

De l'intérêt d'être périphériques

7 En consultant les documents des campagnes électorales de 1989 (élections européennes) et de 1992 (élections législatives britanniques), en interrogeant les responsables des trois partis unionistes (UUP, DUP et Alliance), de l'Orange Order et de l'Ulster Defence Association ${ }^{10}$, on comprend pourtant que cette vision pessimiste du processus de construction européenne est tempérée par un certain réalisme et corrigée par la conscience des avantages afférents à la situation particulière de l'Irlande du Nord. "Nous acceptons le fait que le Royaume-Uni soit membre de la CEE", et nous reconnaissons "qu'il est prudent de participer aux débats internes de ses institutions"; "l'isolationnisme des Conservateurs (britanniques) pourrait faire perdre des occasions à l'Irlande du Nord"; "nous croyons à la coopération avec l'Europe" ; "la Convention européenne des droits de l'homme devrait être incorporée à la Loi fondamentale en Irlande du Nord". Ces commentaires, concédés comme à regret, montrent que dans deux domaines au moins, les responsables unionistes reconnaissent les avantages de l'appartenance à la CEE : le domaine financier et, dans une moindre mesure, celui de la protection des droits individuels. Cette reconnaissance et les conséquences pratiques qui en découlent contribuent à façonner une vision européenne moins négative chez les Unionistes.

8 Cette vision est premièrement celle de l'appartenance de leur province à la catégorie des périphéries de l'Europe, c'est-à-dire de ces régions où la pénétration capitaliste du centre a souvent un effet déstructurant plus puissant que l'effet stimulant de l'ouverture des marchés. Même si depuis l'élargissement de la Communauté en 1986 l'Irlande du Nord n'est plus deuxième (sur 131) sur la liste des régions les plus défavorisées mais seulement trente-troisième (sur 160), la région s'est 
"désindustrialisée" depuis que le Royaume-Uni a rejoint l'Europe ${ }^{11}$ et le taux de chômage avoisine $20 \%$ malgré l'émigration. Comparé en particulier à celui de la République qui était lui aussi en 1973 très inférieur à la moyenne européenne, le PNB de l'Irlande du Nord n'a cessé de se dégrader et il lui faudrait bénéficier d'une croissance de 5,5\% pendant une dizaine d'années pour rattraper son retard. Mais la conscience amère d'être une région sinistrée ${ }^{12} s^{\prime}$ aiguise d'une réflexion contradictoire. Certes, l'Irlande du Nord est largement plus développée que le centre de la Grèce ou le sud du Portugal, mais en même temps elle supporte beaucoup moins bien que la République le choc de l'intégration. N'est-ce pas précisément en raison des "troubles", qui font fuir investisseurs et diplômés, quand ils ne détruisent pas directement les infrastructures? Faut-il donc choisir la négociation pour harmoniser avec Dublin une politique de développement? Mais si la solution "européenne" d'amarrage à la République prévalait, ne serait-ce pas au bénéfice exclusif de celle-ci? Déjà, sous l'effet des directives de la CEE, le nombre des étudiants "du Sud" dans les universités "du Nord", subventionnées par l'État britannique, a doublé entre 1990-91 et 1991-92. En somme, à quoi bon devenir la "périphérie de la périphérie" ?

9 Le deuxième terme du constat unioniste à propos des avantages européens est plus original, mais aussi plus troublant. Engagés dans la complexité communautaire et dans la compétition inter-régionale, les Nord-irlandais se découvrent entre eux une solidarité d'intérêts et une convergence de vues qui dépassent leurs frontières communautaires. Que ce soit au Parlement de Strasbourg ou face aux fonctionnaires de la Commission, les civil servants de la province, et surtout les trois députés européens, s'efforcent de parler d'une seule voix. Ennemis à Belfast, ils coopèrent quotidiennement à Bruxelles, comme en témoigne le soutien unanime qu'a reçu le nouveau Bureau de l'Irlande du Nord. Personne ne s'étonne de voir le bouillant Ian Paisley mettre entre parenthèses son opposition à la CEE pour siéger au Comité pour l'agriculture du Parlement européen; lui-même le reconnaît, la PAC est en enjeu primordial pour la province ${ }^{13}$ et cette préoccupation le rapproche étroitement de son rival du SDLP. C'est ensemble que les trois députés européens d'Irlande du Nord réclament une aide pour les agriculteurs et exposent la crise du textile dans leur province; ensemble, qu'ils soutiennent l'"opération intégrée" proposée directement par la Communauté à la ville de Belfast en 1980. L'immersion dans un espace de dimension continentale et la nécessité d'initier des stratégies de protection et de négociation ont ainsi pour effet de relativiser les divergences à l'intérieur de l'espace nord-irlandais et de faire ressortir en revanche une communauté de destin. D'où, en troisième lieu, la conscience grandissante des Unionistes de devoir gérer l'appartenance au Royaume-Uni et l'adhésion à la CEE non comme une alternative mais comme une "addition" dont il importe de ne perdre aucun bénéfice. Ainsi, l'organisation paramilitaire qu'est l'Ulster Defence Association, par ailleurs hostile au processus d'unification européenne, reconnait tout le parti qu'elle tire de la législation communautaire sur les droits de l'homme, en faveur de ses militants arrêtés. Et emprisonnés, puisque cette législation s'impose avec succès à l'État britannique, même après un long délai ${ }^{14}$. Même raisonnement au plan financier: si le Royaume-Uni accorde à l'Irlande du Nord des subventions dix fois plus élevées que la $\mathrm{CEE}^{15}$, celle-ci lui promet tout de même près de 100 millions $€$ chaque année à travers les tonds structurels communautaires, les subventions de la PAC et bientôt le fonds de cohésion prévu à Maastricht ${ }^{16}$. L'important, comme l'ont compris les députés européens d'Irlande du Nord qui ont fait dans ce sens le siège du gouvernement Major - avec 
l'appui de la Commission et même de l'opposition travailliste à Westminster- c'est que le Royaume-Uni ne déduise pas de son aide à l'Irlande du Nord les subventions votées à Bruxelles au titre des régions défavorisées; comme ce fut le cas avec l'"opération intégrée" à Belfast, la Commission invente d'ailleurs des formules d'intervention qui lui permettent de contourner Londres et de traiter directement avec les régions, en Irlande du Nord comme dans les Highlands ou le Kent. Pour les hommes politiques et les fonctionnaires unionistes, le passage de la relation bilatérale entre la province et le Royaume-Uni à une relation à trois avec la CEE offre, comme à leurs émules nationalistes, l'opportunité d'élargir les ressources de l'Irlande du Nord. Et, comme ces derniers, ils ne la négligent pas. Mais cette nouvelle dimension européenne a pour eux une autre conséquence : la remise en cause de leur loyauté à l'État britannique qui était le fondement même de leur séparation d'avec le Free state, puis de la République du Sud.

Un désir d'Etat

10 Car c'est bien à la période de la Première Guerre mondiale et du partage dé l'île qu'il faut remonter pour appréhender le conflit nord-irlandais dans sa réalité politique et le dégager d'une dimension mythico-religieuse écrasante. En 1920, les Protestants nordirlandais qui avaient contribué héroïquement à la bataille de la Somme trois ans plus tôt ont fait le choix de rester rattachés à un glorieux Empire, loyaux à la Couronne, unis sous la bannière de l'Union Jack. Pour eux, l'appartenance de l'Irlande du Nord au Royaume-Uni signifiait que la province acceptait de se soumettre au centre dans des domaines aussi importants que les relations extérieures, la défense et les finances. Plus tard, le renforcement de cette dépendance par l'imposition d'une administration directe par Londres en 1972 fut un moindre mal, à leurs yeux, qu'une autonomie impliquant un partage du pouvoir avec les Nationalistes.

11 Nul doute que dans l'attachement farouche des Unionistes au Royaume-Uni, est entrée une part croissante d'intérêt bien compris, puisque du welfare state de 1949, aux subventions régionales de Westminster et à la présence de l'armée britannique dans la province qui fait vivre plus de $15 \%$ des familles, l'Irlande du Nord fonctionne comme une entité économique assistée, dont l'abandon par Londres provoquerait le naufrage : ni la république d'Irlande, aux prises avec son propre retard de développement, ni la CEE, nous l'avons $\mathrm{vu}$, ne sauraient pallier la suspension de l'importante aide britannique. Mais il y a bien plus dans la loyauté des Unionistes : la reconnaissance du cadre institutionnel britannique comme celui de leur liberté (même et surtout si cette liberté est au prix de la limitation de celle des Nationalistes) et de leur indépendance, puisqu'à l'attachement à la souveraineté britannique fait écho dans leur conscience la préservation de leur singularité par rapport à la République du Sud. Musique, parades, slogans, peintures murales dans une débauche de rouge et de bleu viennent à intervalles réguliers confirmer et conforter un enthousiasme pour le Royaume-Uni qui n'a guère d'équivalent dans la "grande île". Et leur appréhension de l'ordre international comme un ordre étatique leur paraitt paradoxalement confirmée par les événements mondiaux récents : devant les incertitudes suscitées par la fin de l'Empire soviétique et les bouleversements géopolitiques en Europe méridionale, il leur paraît plus que jamais nécessaire de se rattacher à un État fort.

Or il se trouve que i'État britannique répond de moins en moins à l'idéal des Unionistes, et que son attachement à l'Irlande du Nord évolue à l'inverse de celui que lui témoigne la province. Ce constat est embarrassant, voire déstabilisant pour les Unionistes : le 
Royaume-Uni manifeste depuis le début des "troubles" une tendance de plus en plus claire à se débarrasser du fardeau financier, moral et stratégique que représente l'Irlande du Nord. De plus en plus rares sont les Britanniques qui supportent sans broncher les dépenses civiles et militaires qu'entraînent le maintien de l'ordre et la gestion de la province. Et infime est le nombre de ceux qui acceptent de voir des soldats y perdre la vie. Londres cherche à régler le problème de l'Irlande du Nord dans un sens qui allège sa responsabilité et son fardeau. La solution, plus ou moins explicitement formulée par les gouvernements britanniques, surtout depuis la signature de l'Accord anglo-irlandais par Margaret Thatcher 1985, est celle d'une cogestion au niveau international garantissant un gouvernement de "power sharing" au niveau local. En somme, l'internationalisation, non du conflit, mais de sa solution. Sous cette formule serait délégitimée la revendication nationaliste d'abrogation de la frontière des six comtés tandis que les Unionistes resteraient assurés de la protection de Londres.

Bien entendu, le gouvernement anglais adopte là une stratégie d'État et de négociations bilatérales avec un autre État souverain stratégie que ne manquent pas d'ailleurs de dénoncer les Unionistes. Pour la mettre en oeuvre, le processus de construction européenne lui sert à la fois de masque et d'échappatoire : car tout en se situant au niveau étatique et bilatéral, son approche du problème nord-irlandais est facilitée et stimulée par l'introduction dans la vie politique britannique, irlandaise et nordirlandaise, de la dimension européenne, en particulier au plan juridique, depuis 1973. En relativisant la souveraineté britannique (malgré les fermes précautions que prend Londres à ce sujet) et en facilitant l'introduction de la République dans les discussions sous couvert d'innovation constitutionnelle (comme par exemple dans le projet d'exécutif tricéphale évoqué plus haut), la Communauté européenne interfère dans la relation dyadique entre la province et le Royaume-Uni, une relation que les Unionistes se plaisaient à considérer comme inaliénable. Désormais, le problème d'Irlande du Nord n'est plus l'affaire exclusive du Royaume-Uni : il est tout autant celui de la république d'Irlande, à la fois comme État souverain dans l'île et comme État membre de la CEE.

Les Unionistes comprennent progressivement, à travers la tentative d'imposition du "power sharing" en 1974, l'accord anglo-irlandais de 1985 et l'ouverture en 1991 de négociations à l'initiative du gouvernement de Londres, que le Royaume-Uni n'est plus "leur" État indéfectible et irremplaçable, celui pour lequel leurs pères sont morts en 1917, celui qui ne céderait pas un pouce de souveraineté sur la province. Et ils réagissent sous l'influence d'une autre idéologie dominante en Europe dans Ies années 1980, complémentaire de la construction communautaire, celle du désir d'un État souverain qui coïnciderait avec les aspirations et avec le bien-être d'un peuple. Dès l'origine en 1920, le système constitutionnel nord-irlandais était d'ailleurs porteur d'ambiguïté, qui appelait "État" cette province dotée d'un gouvernement autonome et d'un Parlement local. Et cette ambiguïté n'était pas sans nourrir la vision conservatrice et dominatrice des Protestants puisque cet "État", qui était exclusivement leur, s'est superposé et confondu pendant près de cinquante ans avec l'État britannique dont ils se voulaient loyaux sujets. Mais après l'éclatement des "troubles" et l'arrivée des troupes britanniques, l'ambiguïté est levée. Pour paraphraser une formule célèbre, certains responsables protestants envisagent alors le futur sous la forme d'un État indépendant d'Irlande du Nord - ou mieux, d'Ulster (c'est-à-dire des neuf comtés que comptait traditionnellement la province et non de six seulement) - dans le cadre de l'Europe "des patries"17. Ces hommes et les forces politiques qu'ils représentent sont qualifiés de "Loyalistes", mais si leur loyauté affichée les rattache à la Couronne, leur 
loyauté profonde va à l'Ulster. En mars 1979, le mot indépendance apparaît dans le manifeste du New Ulster Political Research Group, "Beyond the religious divide". Il est repris par l'Ulster Independence Committee et largement publicisé par les dirigeants de l'Ulster Defence Association de l'époque, Tommy Lyttle et Glenn Barr. Mis en avant en 1983 durant la campagne électorale de l'Ulster Loyalist Democratic Party, il ne cessera plus d'être revendiqué par une partie des Protestants de la province.

Depuis 1987 pourtant, l'UDA a officiellement abandonné l'objectif d'indépendance de l'Ulster, et préconise à la place une très large autonomie, le partage des pouvoirs entre Unionistes et Nationalistes, et le maintien d'un lien constitutionnel avec le RoyaumeUni (afin d'écarter les menaces de réunification de l'île) ${ }^{18}$. Cette position de principe est aussi, à des degrés divers, celle de tous les partis unionistes qui sont conscients, à mesure que les négociations anglo-irlandaises progressent, des concessions qu'il leur faut faire aux exigences de Londres pour ne pas avoir à céder à celles de Dublin. De plus, l'expérience communautaire leur apprend à relativiser leur problème et à modérer leurs aspirations : l'Irlande du Nord avec ses quelque $4000 \mathrm{~km} 2$ et son million et demi d'habitants n'est même pas une région au sens où l'entend la CEE, à l'instar de la Catalogne ou de la Lombardie. Comment pourrait-elle raisonnablement fonctionner en État indépendant? La gestion de ses affaires publiques, comme les transports ou l'aménagement du territoire ou la politique de l'emploi, ne peut être envisagée qu'au niveau d'un espace plus vaste, qui est logiquement celui de l'île d'Irlande toute entière, comme en témoigne l'octroi récent par la CEE d'une subvention pour la modernisation de la ligne ferroviaire reliant Belfast à Dublin. Et l'autorité régionale avec laquelle la Commission de Bruxelles est de plus en plus fréquemment amenée à négocier est tout bonnement la République du Sud.

Prendre les armes?

16 Inévitablement, la contradiction entre la loyauté envers le Royaume-Uni et l'aspiration à un État indépendant en Ulster conduit à une rupture au sein de la majorité protestante d'Irlande du Nord. Les réalités prennent la mesure du désengagement britannique et de la nouvelle dimension européenne de l'avenir politique de leur province. Ils observent avec clairvoyance les passions nationalitaires déchaînées dans l'ex-Yougoslavie, jugent avec sévérité l'immobilisme de la CEE et les tentatives de compromis négociées par Lord Carrington (celui-là même qui a "perdu" la Rhodésie, font-ils remarquer), évaluent cyniquement les faibles chances d'un Ulster indépendant menacé d'ostracisme. Indirectement, les événements de $1991-92$ en Europe méridionale et orientale ont donc contribué à débloquer le processus de négociations sur l'Irlande du Nord. Désormais, Alliance, UUP et DUP participent aux réunions de Londres, de Belfast et de Dublin, sur l'avenir de la province; ils acceptent de discuter les propositions de Bruxelles. Ce faisant, les trois partis reflètent les positions de la très large majorité des Protestants nord-irlandais, au point qu'un extrémiste comme le pasteur Paisley, menacé de marginalisation, a dû se résoudre en juillet 1992 à s'asseoir à la même table que le SDLP puis à la même table que le représentant de la République, quelques semaines après avoir proclamé qu'il ne le ferait jamais.

Face à eux, une petite minorité ${ }^{19}$ d'utopistes maintient le flambeau de l'indépendantisme. En réponse aux mots d'ordre des Nationalistes ("troops out' et 'un seul État, la République'), elle se proclame décidée à se battre en 1993 sous le mot d'ordre de 1689 , no surrender !20 Même si l'utilisation du mot colonialisme pour qualifier la présence protestante dans l'île est largement un abus idéologique, la 
comparaison de la situation des Protestants nord-irlandais avec celle des pieds-noirs d'Algérie ${ }^{21}$ dans les années qui précédèrent les accords d'Évian est éclairante. A mesure que Londres prend des décisions qui ouvrent la voie à la cogestion avec la République, telles que l'intégration à l'armée de l'Ulster Defence Regiment en juin 1992, se développent dans leurs cercles Ies thèmes de trahison par la métropole, de capitulation, d'humiliation ${ }^{22}$. Le Royaume-Uni est vilipendé pour son attentisme envers les "Provos" (l'IRA provisoire) à I'heure même où l'effondrement du marxismeléninisme en Europe promettait de ruiner le crédit - du moins le croient-ils - de cette organisation "terroriste".

II est difficile, car ils n'ont guère eu l'occasion de faire leurs preuves, sinon en assassinant des dizaines de Catholiques et en couvrant un efficace réseau de racket, d'évaluer le nombre l'équipement et le niveau d'entraînement des "paramilitaries" protestants qui préparent le "doomsday", le jour où les Ulstériens affronteront l'IrA et l'armée de la République sans le secours des troupes britanniques ${ }^{23}$. Jusqu'à sa tardive interdiction en août 1992 l'Ulster Defence Association servait d'ombrelle à une large diversité de groupuscules tels que l'Ulster Volunteer Force ou les Ulster Freedom Fighters; mais ces combattants ont toujours des ramifications et des appuis dans tous Ies partis protestants, dans l'Orange Order et même dans le Royal Ulster Constabulary, la police locale, et dans l'armée britannique ${ }^{24}$. On sait aussi qu'ils ont obtenu des soutiens à l'étranger, par exemple de l'Afrique du Sud qui leur a procuré des armes par l'intermédiaire d'Israël et des milices chrétiennes du Liban ${ }^{25}$. D'autre part, ils ont cherché à coopérer avec le National Front anglais, Ordine Nuevo en Italie et les Loups Gris de Turquie ${ }^{26}$. A l'instar de l'OAS, ces paramilitaires sont peut-être capables d'entrainer un jour une partie de la communauté protestante dans l'aventure de l'insurrection, dont celle-ci serait, à n'en pas douter, la principale victime, et qui provoquerait leur exode massif vers l'Angleterre et l'Écosse. I1 paraît beaucoup moins probable, mais pas impossible, qu'ils parviennent un temps à contrôler un Ulster indépendant et loyaliste, vidé de ses populations catholiques, sur une partie seulement des six comtés d'Irlande du Nord, consacrant par la même occasion la partition de Belfast: la fièvre nationalitaire et le virus de "libanisation" sont décidément fort contagieux dans l'Europe de cette fin de siècle.

La quête identitaire

19 Le scénario conduisant à une indépendance de l'Ulster apparaît d'autant moins vraisemblable que l'armée britannique est présente en Irlande du Nord (15 000 recrutés locaux, environ 10000 hommes de la grande île) pour lutter contre le terrorisme de l'IRA, donc, indirectement, pour rassurer les Unionistes et les amener à accepter une solution négociée avec la République. Reste que la mise en place d'une autonomie, organisée par un accord anglo-irlandais et garantie par la CEE, ne suffira pas à résoudre le dilemme identitaire des Protestants d'Irlande du Nord. Rien ne les prépare en effet à consoler leur identité britannique blessée en se drapant dans la bannière bleue aux étoiles dorées. Utilitairement, ils savent la valeur de l'Europe ; pratiquement, ils ont commencé à voyager vers le sud et, comme les Anglais, ils prennent leurs congés payés sur la Costa Brava et considèrent les restaurants de pizza comme le summum de la cuisine étrangère. Mais ils n'ont pas renoncé aux stéréotypes qui témoignent d'une immense distance culturelle et affective: "Ulster wheezes in European smog. Sucking polluted air towards Ulster from the continent", titrait par exemple le Belfast 
Telegraph du 23 janvier 1992, comme si sans ce voisinage détestable, le temps serait délicieux en plein hiver au bord de la mer d'Irlande.

A l'Europe trop latine et trop catholique, les Unionistes préfèrent leur identification au monde anglo-saxon et protestant, celui des Pays-Bas de Guillaume d'Orange, de l'Allemagne de Luther, et surtout des Etats-Unis dont ils se plaisent à répéter que leurs exilés ont conduit avec succès la Révolution et la guerre d'indépendance, enfin

celui des bastions presbytériens de l'ancien Commonwealth, de l'Ontario à la province du Cap. Ils font surtout référence à une autre communauté culturelle, celle qui les lie à une Écosse et un pays de Galles en pleine effervescence nationaliste, au point que certaines paroisses presbytériennes des comtés de Down et d'Antrim revendiquent leur appartenance à une "nation écossaise". Dans ces "franges celtiques" du Royaume-Uni, les populations sont d'autant plus séduites par les sirènes du régionalisme, et tentées par un projet nationalitaire ${ }^{27}$, que la prospérité relative et la morgue des Anglais de Londres et du Sud-Est leur paraissent insultantes, mais aussi que la CEE encourage les manifestations d'identité régionale en se plaisant à traiter directement avec les pouvoirs locaux et à court-circuiter des Etats jaloux de leur souveraineté. Cependant, le résultat des Législatives de 1992, en préparation desquelles les indépendantistes d'Écosse, du pays de Galles et d'Irlande du Nord s'étaient concertés, incitent à la prudence. En Écosse, on a vu qu'il y a loin de la "dévolution" à l'indépendance ${ }^{28}$. Et le "break-up" de la Grande-Bretagne, annoncé par T. Nairn en $1981^{29}$, n'est pas prêt de conduire à la naissance d'une "Fédération des îles britanniques".

Justement, l'allusion à une telle Fédération, couplée à celle d'une "frange celtique", souligne les contradictions de la revendication identitaire des Protestants loyalistes. Car la culture celtique à laquelle font référence Écossais, Gallois et Ulstériens pour marquer leur différence par rapport au Royaume-Uni est également - on pourrait même dire spécifiquement - la culture de la population de l'Eire, celle qui fonde, pourrions-nous dire, l'État-nation irlandais. Comment justifier alors le maintien d'une frontière entre la République et l'Irlande du Nord? Un pédiatre proche de I'UDA a "inventé" il y a une dizaine d'années I'histoire des Cruthins, ancêtres des Nordirlandais actuels (protestants et catholiques), qui auraient peuplé l'Ulster et l'ouest de l'Écosse plusieurs siècles avant les Celtes ${ }^{30}$. Comme tant de "nations" d'Europe le font et s'apprêtent à le faire, les Nord-irlandais seraient ainsi fondés à réclamer un État indépendant. Plus sérieusement, l'émergence du régionalisme celtique et son inscription dans l'espace de la CEE se conjuguent pour délégitimer le partage de l'île d'Irlande, tant au plan culturel qu'au plan économique et sous l'angle de la démocratie ${ }^{31}$.

Pour les Unionistes, les perspectives d'avenir sont peu réjouissantes. Dans l'Europe de demain, organisée en régions autour d'un centre marchand dynamique, ils deviendront la périphérie (protestante) de la périphérie (nord-irlandaise) d'une périphérie (l'Irlande). Ils ne sont pas seulement menacés de perdre leur position dominante, et les avantages qu'elle leur valait, ils risquent de devenir progressivement et malgré eux, étrangers à l'État qui structurait leur horizon politique et moral, au point de recourir à des choix extrêmes, émigrer et prendre les armes. Et la logique économique qui se met en place en Europe ne fera rien pour apaiser leurs frustrations nationalitaires. 


\section{NOTES}

1. Le oui a obtenu $52,1 \%$ au référendum de juin 1975 , et le taux de participation n'était que de $48 \%$. Cf. P. Joannon, "The Unionist Fig Leaf or the Dilemma of the Modern Ulster Protestant", Etudes Irlandaises, vol ; XVI, n² 2, décembre 1991, p. 149.

2. Dans son programme électoral pour les législatives britanniques d'avril 1992.

3. E. Moxon-Browne mentionne la rupture de l'union monétaire anglo-irlandaise, les taux différents de TVA, les systèmes différents de sécurité sociale, les taux différents de subvention incitant à un développement de la contrebande de part et d'autre d'une frontière dont l'existence se renforce. E. Moxon-Browne, The Impact of the European Community, in Northern Ireland: Politics and the Constitution, ed. par B. Hadfield, Buckingham, Open University Press, 1992, p. 49.

4. Parlement européen, report Drawn up on Behalf of the Political affairs committee on the situation in Northern Ireland, 1984, doc. 1-152/83, sous la direction de N. Haagerup. 5. Son prédecesseur de 1979 à 1989, John Taylor, avait démissionné de ce groupe pour protester contre l'accord anglo-irlandais et s'était affilié, en 1987, au groupe des droites européennes.

6. En 1991, 68\% de la population d'Irlande du Nord estime que la province devrait rester partie prenante du Royaume-Uni. Cf. A. Guelke, F. Wright, "On a " British Withdrawal" from Northern Ireland", in P. Stringer, G. Robinson (eds.), Social Attitudes in Northern Ireland, the second report 1991-1992, p. 41.

7. Centre for the Study of Conflict, University of Ulster, A north Ireland Centre in Bruxelles, a feasibility report, Corelaine, s. d., 1991, 32 p.

8. Irish Times, 10/07/1992, "EC Officials Worked on SDLP Models".

9. New Ulster defender, vol. 1, n², 1992, Euopean Influences on Ulster's Furture". 10. $L$ 'Orange Order encadre la vie religieuse et culturelle des Protestants, en relation avec les partis unionistes et les milices protestantes ; L'Ulster Defence Association qui fédérait des organisations locales d'entraide et d'autodéfense servait d'ombrelle à des organisations paramilitaires (UFF, UVF) responsables d'assassinats et de rackets; elle a été déclarée hors la loi le 10/08/1992. Interviews à Belfast du 1er au 10 juillet 1992, au cours d'une mission du Centre d'Etudes et de Recherches Internationales.

11. La production industrielle aurait décrue de 1,1\% par an entre 1971 et 1986 selon R. Harris, Regional Economic Policy in Northern Ireland 1945-1988, Aldershot, Gower Publishing cy, 1991, p. 107.

12. B. Feeney, "A peripheral concern ?", Fornight, novembre 1990.

13. Belfast Telegraph, 17 janvier 1992.

14. Ulster defence Association, A Bill of Rights, Belfast, 1986.

15. E. Moxon-Browne, op. cit., p. 56. Selon B. Crick "The Question questioned", The Irish review, 8, printemps 1990, p. 95, chaque Irlandais du Nord bénéficie environ de $1000 £$ par an de subventions extérieures.

16. Business Telegraph, 11 février 1992 ; Irish News, 13 février 1992.

17. Jacques Leruez explique très bien à propos des nationalistes écossais combien le mot d'indépendance dans l'Europe permet au SLP de récuser à la fois l'accusation de séparatisme et celle de républicanisme. Cf. J. Leruez, "Le parti national écossais et l'Europe", annales de l'Université de Savoie, n 14, 1991, pp. 3-14. 
18. Common Sense, rédigé sous la direction de John McMichael et publié par l'Ulster Political Research Group.

19. Qui ne serait que de $1 \%$ d'après les chiffres de l'enquête de 1991 analysée par $A$. Guelke et F. Wright, op. cit. p. 41.

20. assiégés durant l'hiver 1688-1689, les Protestants de Londonderry accueillirent les troupe du roi catholique Jacques II par ce cri.

21. Elle a été proposée en particulier par F. Wright, "Is Northern Ireland Britain's Algeria ?", Etudes Irlandaises, vol. XVI, nº 2, décembre 1991, pp. 115-131.

22. Cf. Ulster New Defender, op. cit.

23. A. Aughey, D. Hume, "Delopments Within the UDA : A New Paramilitary Emphasis ?", Teaching Politics, vol. XV, n 2, mai 1986, pp. 315-387.

24. Le Monde, 23 septembre 1989, "Prouvée par toute une série de documents, la collusion entre services de sécurité et groupes paramilitaires protestants se confirme", Just News, vol. V, n 10 (1990) ; Le Monde, 5 février 1992, The Independent, 30 juillet 1992.

25. A. Guelke, "British Policy and International Dimensions of the Nortjern Ireland Conflict", Regional Politics and Policy, vol. I, n 2, 1991, p ; 154.

26. C. Johnson, "The national Front and the Ulster Connection", Fornight, juilletseptembre 1986, pp. 25-32.

27. J. Foster, "Radical Regionalism, The Irish Review, $\mathrm{n}^{\circ}$ 7, automne 1989, pp. 1-15.

28. J. Leruez, L'Ecosse, une nation sans Etat, Lille, Presse Universitaures de Lille, 1983.

29. T. Nairm, The break-up of Britain. Crisis and Neo-Nationalism, Londres, Verso, 1981.

30. I. Adamson, The Identity of Ulster, the Land, the Language and the People, Belfast, Pretami Press, 1991. Cf. aussi une série d'articles publiés par Ulster Magazine entre février et août 1990. Sur la manipulation de l'histoire à des fins nationalistaires, cf. E. Picard, "Milices libanaises et paramilitaires irlandaises : de la mobilisation du groupe à l'invention de son identité, texte multigr., Paris, CERI, 1992.

31. B. O'Seaghdha, "Ulster Regionalism : the Unpleasant facts", The Irish Review, 8, printemps 1990, p. 58.

\section{RÉSUMÉS}

La question d'Irlande du Nord est aussi celle de l'avenir des Protestants (60 \% des habitants de la province), dont la méfiance, voire l'hostilité, envers la construction européenne est l'image inversée des attentes et des satisfactions des Catholiques. Isolés à Bruxelles et à Strasbourg, les élus protestants découvrent leur communauté d'intérêt avec les Catholiques pour réclamer un soutien de la CEE à leur province qui s'ajoute, et ne se substitue pas, à l'aide britannique. Pourtant, devant le glissement du problème de l'Irlande du Nord de l'espace britannique à l'espace européen, une minorité indépendantiste prône la lutte armée. La fièvre nationalitaire enflamme aussi cette périphérie de l'Europe.

The Northern Ireland problem is closely linked to that of the future of its Protestant population (60\%), profoundly suspicious and even hostile to Europeanism, in complete contrast to the attitude of the Catholics. Feeling isolated in Brussels and Strasbourg, the Protestant delegates 
discover a community of interest with their Catholic countrymen in claiming European aid for their province, in addition to what they already receive from the United Kingdom. Nonetheless, despite a gradual displacement of the Northern-Irish problem from the British to the European level, a minority of independence extremists still advocate for an armed struggle. Nationalist fever continues inflaming this periphery of Europe.

\section{INDEX}

Index géographique : Europe, Irlande du Nord, Royaume Uni

Mots-clés : religion, violence, identité

\section{AUTEUR}

\section{ELISABETH PICARD}

Chercheur au Centre d'Etudes et de Recherches Internationales, Maître de Conférences à l'Institut d'Etudes Politiques de Paris, spécialiste du monde arabe. 